\title{
Infusão venosa aquecida relacionada à prevenção das complicações da hipotermia intraoperatória*
}

\author{
Warmed venous infusion in the prevention of intraoperative hypothermia complications \\ Infusión venosa calentada relacionada con la prevención de las complicaciones de \\ hipotermia intraoperatoria
}

\author{
Nathália Haib Costa Pereira ${ }^{1}$, Adelaide De Mattia Rocha², Ana Lúcia De Mattia ${ }^{3}$
}

RESUMo: Objetivo: Analisar as complicações relacionadas à hipotermia no período intraoperatório, na utilização da infusão intravenosa aquecida. Método: Estudo experimental, comparativo, de campo, prospectivo e quantitativo, desenvolvido em um hospital público federal. A amostra foi constituída por 60 adultos, sendo um dos critérios de inclusão a temperatura axilar estar entre $36^{\circ} \mathrm{C}$ e $37,1^{\circ} \mathrm{C}$; esses indivíduos foram divididos em grupos controle e experimental, compostos utilizando-se a técnica de amostragem probabilística sistemática. Resultados: As complicações apresentadas foram: taquicardia, hipertensão arterial e sangramento. A hipertensão arterial e o sangramento apresentaram significância estatística entre os grupos, com $p=0,0150$ e $p=0,0050$, respectivamente. A taquicardia não apresentou significância estatística entre os grupos $(\mathrm{p}=1,0000)$. Conclusão: A utilização da infusão venosa aquecida isolada não previne as complicações relacionadas à hipotermia intraoperatória. Compete ao Enfermeiro o planejamento das intervenções de Enfermagem para prevenção da hipotermia e manutenção da normotermia.

PALAVRAS-CHAVE: Hipotermia. Enfermagem perioperatória. Complicações intraoperatórias.

TRIAL: (REQ:1453).

ABSTRACT: Objective: The present study aims to analyze the complications related to hypothermia in the intraoperative period using warmed venous infusion. Method: An experimental, comparative, prospective, field study using a quantitative approach was carried out in a public hospital. The sample consisted of 60 adults divided into one control and one experimental group. The groups were formed using the systematic probabilistic sampling technique. Inclusion criteria comprised axillary temperature between 36 and $37.1^{\circ} \mathrm{C}$. Results: The following complications were observed: tachycardia, arterial hypertension, and bleeding. Arterial hypertension and bleeding presented statistical significance between the groups, $p=0.0150$ and $p=0.0050$, respectively. No statistical significance was found between the groups $(p=1.0000)$ for tachycardia. Conclusion: The use of isolated warmed venous infusion does not prevent complications related to intraoperative hypothermia. It is the nursing staff's duty to plan the nursing interventions required to avoid hypothermia and maintain normothermia.

KEYWORDS: Hypothermia. Perioperative nursing. Intraoperative complications.

TRIAL: (REQ:1453).

RESUMEN: Objetivo: Analizar las complicaciones relacionadas con la hipotermia en el período intraoperatorio cuando se utiliza infusión intravenosa calentada. Método: Estudio experimental, comparativo, de campo, prospectivo y cuantitativo, desarrollado en un hospital público federal. Se eligió una muestra de 60 adultos, siendo uno de los criterios de inclusión la temperatura axilar entre $36^{\circ}$ y $37,1^{\circ} \mathrm{C}$, divididos en grupos control y experimental a través de la técnica de muestreo probabilístico sistemático. Resultados: Las complicaciones verificadas fueron: taquicardia, hipertensión arterial y sangrado. La hipertensión arterial y el sangrado presentaron significancia estadística entre los grupos, con $\mathrm{p}=0,0150$ y $\mathrm{p}=0,0050$, respectivamente. La taquicardia no presentó significancia estadística entre los grupos $(\mathrm{p}=1,0000)$. Conclusión: La utilización de infusión venosa calentada aislada no previene las complicaciones relacionadas con la hipotermia intraoperatoria. Incumbe al enfermero la planificación de las intervenciones de enfermería para la prevención de la hipotermia y el mantenimiento de la normotermia.

PALABRAS CLAVE: Hipotermia. Enfermería perioperatoria. Complicaciones intraoperatorias.

TRIAL: (REQ:1453).

${ }^{1}$ Graduanda em Enfermagem da Universidade Federal de Minas Gerais - UFMG. E-mail: nathaib@hotmail.com

${ }^{2}$ Enfermeira. Doutora em Enfermagem. Professora Associada do Departamento de Enfermagem Básica da Escola de Enfermagem da Universidade Federal de Minas

Gerais - UFMG. E-mail: adelaidedemattia@gmail.com

${ }^{3}$ Enfermeira. Doutora em Enfermagem. Professora do Departamento de Enfermagem Básica da Escola de Enfermagem da Universidade Federal de Minas Gerais - UFMG.

Orientadora da pesquisa.

Rua Aquiles Lobo, 314 apto. 04. Floresta. CEP 30150-160. Belo Horizonte, MG, Brasil.

Telefone: (31) 3409-9886. E-mail: almattia@uol.com.br

* Pesquisa com apoio financeiro da Fundação de Amparo à Pesquisa do Estado de Minas Gerais (FAPEMIG). 


\section{Introdução}

A hipotermia é um dos diagnósticos de Enfermagem mais frequentemente encontrados em pacientes na sala de recuperação pós-anestésica, sendo suas causas provenientes do período intraoperatório ${ }^{1}$. É um estado clínico de temperatura corporal abaixo do normal (inferior a $36^{\circ} \mathrm{C}$ ), sendo classificada como leve, entre $35,9^{\circ} \mathrm{C}$ e $34^{\circ} \mathrm{C}$; moderada, entre $33,9^{\circ} \mathrm{C}$ e $30^{\circ} \mathrm{C}$; grave, inferior a $29,9^{\circ} \mathrm{C}^{2}$.

Uma das principais causas da hipotermia é a baixa temperatura mantida na sala de operação $(\mathrm{SO})$, entre $19^{\circ} \mathrm{C}$ e $24^{\circ} \mathrm{C}$, preconizada pelo Ministério da Saúde (MS), além de outros fatores, como: cirurgias abdominais e torácicas, em que as cavidades corporais permanecem abertas e expostas às baixas temperaturas; infusão intravenosa de líquidos e hemoderivados frios; uso de soluções para irrigação e preparações para a pele frias; tempo de duração da cirurgia, e agentes anestésicos ${ }^{3,4}$. Também pode ser desencadeada por fatores de risco que predispõem o paciente a desenvolver a hipotermia, como extremo de idade, morfologia corporal, estado pré-operatório e condições pré-existentes, como distúrbios neurológicos, doenças crônicas e traumas ${ }^{4-6}$.

Medidas de aquecimento ativo incluem infusão venosa aquecida, irrigação de fluidos aquecidos, uso de colchões de água circulante aquecida e calor radiante, que podem manter a normotermia, quando utilizadas isoladamente ou em combinação com o aquecimento de ar forçados.

A hipotermia é comum e causa complicações, tais como coagulação e alterações da função plaquetária, aumento da morbidade cardíaca e infecção de sítio cirúrgico, bem como eleva os níveis de incidência de úlcera por pressão ${ }^{7}$.

Embora a hipotermia seja uma complicação comum no período perioperatório, a escassez de trabalhos sobre essa temática é evidenciada em um estudo realizado no ano de 2008, por levantamento bibliográfico, o qual apontou que, dos 297 periódicos analisados, apenas quatro estudos tratavam da hipotermia ${ }^{8}$.

Os estudos tratam a hipotermia como uma complicação perioperatória. Esta pesquisa tem como finalidade a análise das complicações intraoperatórias ocasionadas pela hipotermia. Desta forma, levanta-se a seguinte questão: a infusão venosa aquecida previne as complicações relacionadas à hipotermia intraoperatória?

\section{Objetivo}

Analisar as complicações relacionadas à hipotermia no período intraoperatório, na utilização da infusão intravenosa aquecida.

\section{Método}

Trata-se de um estudo com abordagem metodológica quantitativa, delineamento experimental, comparativa, de campo e prospectiva. Em um estudo experimental, o investigador manipula a variável independente, administrando um tratamento experimental (ou uma intervenção experimental) a alguns sujeitos, ao mesmo tempo em que não o faz a outros 9 .

O estudo foi realizado no centro cirúrgico de um hospital público e universitário, na cidade de Belo Horizonte-MG. Foram selecionadas duas SO para o estudo, por apresentarem características semelhantes, no que se refere ao atendimento de especialidade de cirurgia do aparelho digestório (CAD), quanto à área, temperatura e umidade ambiental, entre $19^{\circ} \mathrm{C}$ e $24^{\circ} \mathrm{C}$, e 45 e $60 \%$, respectivamente, conforme as recomendações do $\mathrm{MS}^{10}$.

O projeto de pesquisa foi aprovado pelo Comitê de Ética em Pesquisa da Universidade Federal de Minas Gerais (UFMG), atendendo à Resolução 196/1996 (atual Resolução 466/2012) do Conselho Nacional de Saúde (CNS), com o parecer $n^{\circ}$ ETIC 310/09.

O Termo de Consentimento Livre e Esclarecido (TCLE) foi assinado por todos os participantes, após receberem do pesquisador as informações sobre o estudo e seus objetivos. Os esclarecimentos e a assinatura do TCLE foram realizados no quarto do paciente, no dia da cirurgia, antes da administração da medicação pré-anestésica, quando indicada.

O tamanho amostral foi definido segundo o número de variáveis preditivas inicialmente propostas, utilizando-se de cinco a oito sujeitos em relação a cada uma das variáveis inicialmente propostas, perfazendo uma amostra de 60 sujeitos ${ }^{11}$.

A composição do grupo controle (GC) e do grupo experimental (GE) foi realizada utilizando-se a técnica de amostragem probabilística sistemática.

Partiu-se de um sorteio para determinar o grupo do primeiro sujeito componente da amostra, se GC ou GE; esse sujeito foi sorteado para o GE e, a partir deste, o segundo paciente foi para o $\mathrm{GC}$, sendo assim sucessivamente intercalados, até completar 30 pacientes em cada grupo.

Os sujeitos do GE receberam infusão venosa aquecida durante todo procedimento anestésico-cirúrgico e os sujeitos do GC não receberam cuidados específicos para prevenção da hipotermia, conforme os procedimentos da instituição. Todos os sujeitos receberam aquecimento passivo de cobertura com lençol.

Foram critérios de inclusão na amostra: ter assinado o TCLE; ser adulto com idade superior a 18 anos; procedimento cirúrgico eletivo, com acesso cirúrgico abdominal convencional ou mínimo; anestesia geral; tempo anestésico de no mínimo uma hora; classificação física da American Society Anesthesiologists (ASA) de ASA I e ASA II, e temperatura corpórea axilar ao entrar na $\mathrm{SO}$ entre $36^{\circ} \mathrm{C}$ e $37,1^{\circ} \mathrm{C}$.

Excluíram-se pacientes com predisposição às alterações de temperatura, como distúrbios da tireoide e neurológicos, extremos de peso, classificação de ASA III a VI, e temperatura corpórea axilar inferior a $36^{\circ} \mathrm{C}$ ou superior a $37,1^{\circ} \mathrm{C}$, ao entrar na SO. 
$\mathrm{O}$ aquecimento da infusão venosa foi feito por estufa, da marca Fanem, linha 502, versão A, com termostato eletrônico e mantida a $40^{\circ} \mathrm{C}$, propiciando, desta forma, que as infusões se mantivessem em temperaturas entre $37^{\circ} \mathrm{C}$ e $38^{\circ} \mathrm{C}$. Foram feitos testes para a adequação da temperatura da estufa com a temperatura da infusão venosa, com a finalidade de controlar a temperatura de infusão venosa conforme o limite superior da temperatura corpórea considerada normal.

Do paciente, foram coletados dados referentes ao grupo a que pertence (GC ou GE), sexo, idade, classificação de ASA, temperatura corpórea no momento de entrada em SO e as alterações apresentadas pelos pacientes do GC e GE, em SO.

No que se refere ao procedimento anestésico-cirúrgico, foram considerados dados referentes ao tipo de cirurgia realizada, potencial de contaminação do procedimento cirúrgico e tempo de duração da anestesia. O dado ambiental foi a temperatura da $\mathrm{SO}$ na entrada do paciente.

Para verificação da temperatura da $\mathrm{SO}$, foi utilizando o termômetro de marca Thermometer, posicionado a um metro da cabeceira da mesa de operação.

A mensuração da temperatura axilar do paciente ocorreu tanto na entrada como na saída da SO, utilizando-se o termômetro clínico digital Pro Check TH186. A coleta de dados ocorreu entre maio de 2011 e abril de 2012, e foi realizada por um dos pesquisadores.

O software utilizado na análise dos dados foi $\mathrm{R}$, na versão 2.13.1. Para verificar a homogeneidade entre o GC e o GE, foi utilizado o teste de Mann-Whitney, empregado para a comparação das variáveis quantitativas, sendo apresentados os resultados por meio de média aritmética, mediana, valores máximo e mínimo, erro padrão, com nível de significância de $5 \%$.

O teste qui-quadrado foi utilizado para as variáveis qualitativas e o teste Exato de Fisher foi utilizado para estas variáveis, quando os valores esperados da tabela de contingência foram menores do que cinco.

\section{Resultados}

Os resultados estão apresentados com dados relativos à caracterização do paciente e do procedimento anestésicocirúrgico, e à caracterização das temperaturas do paciente, do ambiente e das complicações apresentadas pelos pacientes do GC e GE, no período intraoperatório.

\section{Caracterização do paciente e do procedimento anestésico-cirúrgico}

Em relação ao sexo, houve semelhança entre os grupos, com prevalência do sexo feminino, sendo que $23(76,6 \%)$ e 22 $(73,3 \%)$ eram do sexo feminino e sete $(23,4 \%)$ e oito $(26,7 \%)$ do masculino, no GC e GE, respectivamente $(\mathrm{p}=0,7660)$.

A média de idade dos pacientes no GC foi de 45,4 anos, a mediana foi de 45,5 anos e o erro padrão foi de 2,48 (18-62).
No GE, a média foi de 49,6 anos, a mediana de 54,0 anos e o erro padrão de 2,74 (20-64) ( $\mathrm{p}=0,2608)$.

Houve também semelhança entre os grupos na avaliação da condição física de ASA, com maior frequência de ASA II, sendo 15 pacientes $(68,1 \%)$ no $\mathrm{GC}$ e 13 pacientes $(59,0 \%)$ no $\mathrm{GE}(\mathrm{p}=0,793)$.

O procedimento cirúrgico de maior frequência para os dois grupos foi a colecistectomia laparoscópica, devido à colelitíase, com seis pacientes $(27,2 \%)$ no $\mathrm{GC}$ e cinco pacientes $(22,7 \%)$ no GE, seguido dos procedimentos cirúrgicos de ginecologia, por distúrbios, como endometriose, miomas uterinos, cistos ovarianos, entre outros, com quatro pacientes $(18,1 \%)$ no GC e seis pacientes $(26,6 \%)$ no GE.

Quanto ao potencial de contaminação, os procedimentos classificados como limpos foram 22 e 21 , potencialmente contaminados quatro e seis, contaminados três e três, infectados um e zero, no GC e GE, respectivamente $(\mathrm{p}=0,911)$.

O tempo de duração da anestesia foi semelhante nos grupos. A média de duração da anestesia no GC foi de 183,80 minutos e o erro padrão foi de 14,69 minutos $(60,0-330,0)$. No GE, a média foi de 183,53 minutos e o erro padrão foi de 15,04 minutos $(80,0-400,0)(\mathrm{p}=0,946)$.

\section{Caracterização das temperaturas do paciente, do ambiente e das alterações}

A temperatura mediana dos pacientes na entrada da SO foi de $36,4^{\circ} \mathrm{C}$ no $\mathrm{GC}$ e de $36,1^{\circ} \mathrm{C}$ no GE, sendo essa diferença marginalmente significativa $(p=0,0562)$.

A temperatura da $\mathrm{SO}$, na entrada do paciente, não apresentou significância estatística entre os grupos $(\mathrm{p}=0,1776)$.

Caracterizando a hipotermia como a temperatura do paciente menor ou igual a $36^{\circ} \mathrm{C}$, nesta pesquisa, o mesmo número de pacientes dos dois grupos (GC e GE) apresentou hipotermia na saída da SO, sendo que em $22(73,4 \%)$ ocorreu no GC e em 22 (73,4\%), no GE, $(p=1,0000)$.

A Tabela 1 demonstra que as alterações apresentadas pelos pacientes em SO foram a taquicardia, a hipertensão arterial e o sangramento.

A taquicardia não apresentou significância estatística entre os grupos; a frequência foi de três $(10,0 \%)$ pacientes de cada grupo $(\mathrm{p}=1,0000)$.

Quanto à hipertensão arterial, houve significância estatística entre os grupos $(p=0,0150)$, sendo três $(10,0 \%)$ pacientes do GC e 11 (36,7\%) do GE, conforme Tabela 1.

Observa-se que o sangramento foi apresentado por oito (26,7\%) pacientes do GC e nenhum paciente do GE, havendo significância estatística $(\mathrm{p}=0,0050)$.

A chance de ocorrência de sangramento nos pacientes do GE foi $0,05(0,002-0,79)$ vez a chance do GC, conforme demonstrado na Tabela 1. 
Tabela 1. Caracterização das complicações intraoperatórias apresentadas pelos pacientes do grupo controle (GC) e do grupo experimental (GE). Belo Horizonte-MG, Brasil, 2012.

\begin{tabular}{|c|c|c|c|c|c|c|c|c|c|}
\hline \multirow{2}{*}{\multicolumn{2}{|c|}{ Complicações intraoperatórias }} & \multicolumn{4}{|c|}{ Grupo } & \multirow{2}{*}{ p-valor } & \multirow{3}{*}{$\begin{array}{c}\begin{array}{c}\text { Odds } \\
\text { ratio }\end{array} \\
1\end{array}$} & \multicolumn{2}{|c|}{ IC - 95\% } \\
\hline & & \multicolumn{2}{|c|}{ Controle } & \multicolumn{2}{|c|}{ Experimental } & & & LI & $\mathbf{L S}$ \\
\hline Taquicardia & Não & 27 & $90,0 \%$ & 27 & $90,0 \%$ & \multirow{3}{*}{1,0000} & & - & - \\
\hline & $\operatorname{Sim}$ & 03 & $10,0 \%$ & 03 & $10,0 \%$ & & 0,72 & 0,207 & 4,82 \\
\hline & Total & 30 & $100,0 \%$ & 30 & $53,3 \%$ & & & & \\
\hline \multirow[t]{3}{*}{ Hipertensão arterial } & Não & 27 & $90,0 \%$ & 19 & $63,3 \%$ & \multirow{3}{*}{0,0150} & 1 & - & - \\
\hline & Sim & 03 & $10,0 \%$ & 11 & $36,7 \%$ & & 5,21 & 1,278 & 21,2 \\
\hline & Total & 30 & $100,0 \%$ & 30 & $100,0 \%$ & & & & \\
\hline \multirow[t]{3}{*}{ Sangramento } & Não & 22 & $73,3 \%$ & 30 & $100,0 \%$ & \multirow{3}{*}{0,0050} & 1 & - & - \\
\hline & Sim & 08 & $26,7 \%$ & - & - & & 0,05 & 0,002 & 0,79 \\
\hline & Total & 30 & $100,0 \%$ & 30 & $100,0 \%$ & & & & \\
\hline
\end{tabular}

\section{Discussão}

Os resultados evidenciaram que houve homogeneidade entre os grupos GC e GE, no que se refere a: sexo, idade, classificação de ASA, tipo e potencial de contaminação do procedimento anestésico-cirúrgico, e tempo de duração da anestesia, não apresentando significância estatística entre os grupos.

Neste estudo, foram controladas as variáveis de temperatura do paciente e do ambiente, tanto na entrada quanto na saída da SO. A temperatura do paciente na entrada da SO foi controlada de 36 a $37,1^{\circ} \mathrm{C}$ e, da SO, na entrada do paciente, de 19 a $24^{\circ} \mathrm{C}$. Apenas a temperatura de entrada do paciente em $\mathrm{SO}$ apresentou uma diferença marginalmente significativa entre os grupos $(\mathrm{p}=0,0562)$.

Desta forma, os dois grupos (GC e GE) são estatisticamente homogêneos. A hipotermia instalou-se tanto no GE - que recebeu infusão venosa aquecida durante todo o procedimento anestésico-cirúrgico - quanto no GC, sendo $22(73,4 \%)$ pacientes de cada grupo, com temperatura corpórea abaixo de $36^{\circ} \mathrm{C}$, na saída da SO. As alterações apresentadas, tanto para os pacientes do GC quanto para os do GE, foram a taquicardia, a hipertensão arterial e o sangramento.

A taquicardia não apresentou significância estatística entre o GC e o GE; entretanto, houve significância estatística para a hipertensão arterial e o sangramento.

A hipertensão arterial e a taquicardia se devem ao aumento das catecolaminas durante a hipotermia. Até mesmo a hipotermia leve aumenta o nível sérico de catecolaminas, levando aos quadros de taquicardia, hipertensão arterial e vasoconstrição sistêmica, e ao desequilíbrio entre a demanda e a oferta de oxigênio no miocárdio, além de aumentar a irritabilidade miocárdica ${ }^{12}$.

Nesta pesquisa, a chance (Odds ratio) de os pacientes do GE apresentarem hipertensão arterial, em relação ao GC, foi de 5,21 vezes $(1,27-21,2)$.

As complicações adversas decorrentes da hipotermia intraoperatória são: aumento da incidência de infecção na ferida operatória, decorrente da vasoconstrição subcutânea, gerando hipóxia tecidual; aumento do índice de hemorragia e da necessidade de transfusões sanguíneas; tremores musculares que aumentam o consumo de oxigênio de $400 \%$ a $500 \%$ e aumentam a produção de dióxido de carbono; demandas cardíacas e respiratórias elevadas; prolongamento do efeito de muitas classes de fármacos, aumentando o tempo de inconsciência; consequências miocárdicas; aumento do tempo de permanência na sala de recuperação pós-anestésica, e prolongamento do período de hospitalização ${ }^{4,5,13}$.

O sangramento foi apresentado por oito $(26,7 \%)$ dos pacientes do GC e nenhum paciente do GE, com significância estatística $(\mathrm{p}=0,0050)$, sendo que a chance de ocorrência de sangramento nos pacientes do GE é $0,05(0,002-0,79)$ vezes a chance do GC.

Apesar de a contagem de plaquetas permanecer normal durante a hipotermia, há indução de alterações morfológicas sugestivas de ativação plaquetária. Testes, como o tempo de protrombina (TP) e o tempo de tromboplastina parcial ativada (TTPA), permanecem normais porque são realizados a $37^{\circ} \mathrm{C}$, independentemente da temperatura do paciente. Quando realizados na temperatura em que o paciente se encontra, esses testes se tornam alterados, porque há redução na velocidade das reações enzimáticas da cascata de coagulação. Dois estudos aleatórios controlados confirmaram que a redução da temperatura central $\mathrm{em} 0,5^{\circ} \mathrm{C}$, em pacientes submetidos à artroplastia de quadril sob anestesia regional, está associada à maior perda sanguínea e à maior necessidade de transfusão alogênica ${ }^{12}$.

Em um estudo experimental, obteve-se que a utilização da infusão venosa aquecida isolada não previne a hipotermia perioperatória; esta deve estar associada com medidas de aquecimento do paciente no período pré-operatório e de controle da temperatura ambiente na SO. As variáveis que demonstraram significância estatística no desenvolvimento da hipotermia intraoperatória foram a temperatura do paciente na entrada da $\mathrm{SO}$ e a temperatura da $\mathrm{SO}$ na entrada do paciente ${ }^{14}$. 
Assim, há necessidade de intervenções para prevenção da hipotermia e manutenção da normotermia, tanto no período intraoperatório quanto no período pré-operatório ${ }^{14}$.

A manutenção da normotermia reduz as possibilidades de desenvolver as complicações operatórias e a prevenção da hipotermia se dá por meio dos métodos de aquecimento, que são classificados em passivos e ativos. O método passivo tem baixo custo, limita a perda de calor por radiação e convecção, e consiste em cobrir toda a superfície cutânea possível, utilizando lençois, cobertores, campos cirúrgicos e mantas aluminizadas. Já o método ativo é mais efetivo, pois não só evita a perda de calor como é fonte de calor; ainda, dependendo do dispositivo utilizado, o custo pode ser mais elevado. São estes: colchão térmico, manta aquecida, lâmpadas infravermelhas radiantes, infusão de soluções aquecidas e aquecimento e umidificação dos gases administrados ao paciente ${ }^{6,15,16}$.

A prevenção de complicações inerentes ao procedimento anestésico-cirúrgico consiste em papel crucial do Enfermeiro, o qual é responsável pelo planejamento e pela implementação de intervenções que minimizem os riscos e assegurem privacidade e segurança para o paciente cirúrgico. Neste cenário, compete ao Enfermeiro a implantação de intervenções eficazes que proporcionam a prevenção ou o tratamento da hipotermia e, consequentemente, a diminuição das complicações associadas a este evento ${ }^{17,18}$.

\section{Conclusão}

Esta pesquisa permitiu concluir que o uso da infusão venosa aquecida isoladamente em pacientes no período intraoperatório não previne a hipotermia e as complicações a esta relacionadas.

Demonstrou-se que o mesmo número de sujeitos, tanto do GC quanto do GE, apresentou complicações no período intraoperatório, como taquicardia, hipertensão arterial e sangramento, sendo que esses sujeitos saíram da $\mathrm{SO}$ com temperatura corpórea inferior a $36^{\circ} \mathrm{C}$.

A taquicardia não apresentou significância estatística entre os grupos $(\mathrm{p}=1,0000)$. Quanto à hipertensão arterial e ao sangramento, houve significância estatística entre os grupos, sendo $\mathrm{p}=0,0150$ e $\mathrm{p}=0,0050$, respectivamente; observe-se que a chance de ocorrência de sangramento nos pacientes do GE é $0,05(0,002-0,79)$ vez a chance do GC.

Conclui-se, com esta pesquisa, que a utilização da infusão venosa aquecida isolada não previne as complicações relacionadas à hipotermia intraoperatória. Diante deste fato, compete ao Enfermeiro o planejamento e a implementação das intervenções de Enfermagem para prevenção da hipotermia e manutenção da normotermia, diminuindo a chance de desenvolvimento de complicações relacionadas à hipotermia intraoperatória.

\section{Referências}

1. De Mattia AL, Maia LF, Silva SS, Oliveira TC. Diagnósticos de enfermería de complicaciones en la sala de recuperación anestésica. Enferm Global [Internet]. 2010 [acesso em 2011 Mar. 18];18(1):1-11. Disponível em: http://revistas.um.es/eglobal/ article/view/93601

2. Potter PA, Perry AG. Fundamentos de enfermagem. 6. ed. Rio de Janeiro: Elsevier; 2005.

3. Brasil. Ministério da Saúde. Portaria $\mathrm{n}^{\circ} 1.884$ de 11 de novembro de 1994. Aprova normas técnicas destinadas ao exame e aprovação de projetos físicos de estabelecimentos assistenciais de saúde e revoga a Portaria MS nº 400, de 6 de dezembro de 1977. Diário Oficial da União; Brasília; 15 dez. 1994.

4. Paulikas CA. Prevention of unplanned perioperative hypothermia. AORN J. 2008;88(3):358-68. PMid:18819204. http://dx.doi. org/10.1016/j.aorn.2008.05.020

5. Hooper VD, Chard R, Clifford T, Fetzer S, Fossum S, Godden $\mathrm{B}$, et al. ASPAN's evidence-based clinical practice guideline for the Promotion of perioperative normothermia. ASPAN J. 2009;24(5):271-89.

6. Pagnocca ML, Tai EJ, Dwan JL. Temperature control in conventional abdominal surgery: comparison between conductive and the association of conductive and convective warming. Rev Bras Anestesiol. 2009;59(1):56-66. PMid:19374216. http://dx.doi. org/10.1590/S0034-70942009000100008

7. Poveda VB, Clark AM, Galvão CM. A systematic review on the effectiveness of prewarming to prevent perioperative hypothermia. J Clin Nurs. 2013;22:906-18. PMid:22978458. http://dx.doi. org/10.1111/j.1365-2702.2012.04287.x

8. Gotardo JM, Silveira RCCP, Galvão CM. Hipotermia no perioperatório: análise da produção científica nacional de enfermagem. Rev SOBECC. 2008;13(2):40-8.

9. Polit DF, Beck CT. Fundamentos de pesquisa em enfermagem: avaliação de evidências para a prática de enfermagem. 7. ed. Porto Alegre: Artmed; 2011.

10. Brasil. Ministério da Saúde. Portaria $n^{\circ} 2.616$ de 12 de maio de 1998. Dispõe sobre normas de controle de infecções hospitalares. Diário Oficial da União; Brasília; 13 maio 1998.

11. Chattefuee S, Hadi AS. Regression analysis by example. New Jersey: John Wiley \& Sons; 2006. http://dx.doi.org/10.1002/0470055464

12. Biazzotto CB, Brudniewski M, Schmidt AP, Auler JOC Jr. Hipotermia no período perioperatório. Rev Bras Anestesiol. 2006;56(1):89-106. PMid:19468555. http://dx.doi. org/10.1590/S0034-70942006000100012

13. De Mattia AL, Barbosa MH, Rocha AM, Farias HL, Santos CA, Santos DM. Hypothermia in patients during the perioperative period. Rev Esc Enferm USP. 2012;46(1):60-6. PMid:22441266. http://dx.doi.org/10.1590/S0080-62342012000100008

14. De Mattia AL, Barbosa MH, Freitas JPA, Rocha AM, Pereira NHC. Infusão venosa aquecida no controle da hipotermia no período intraoperatório. Rev. Latinoam. Enferm. 2013;21(3):803-10.

15. Tramontini CC, Graziano KU. Controle da hipotermia de pacientes cirúrgicos idosos no intraoperatório: avaliação de duas intervenções de enfermagem. Rev Latino-Am Enferm. 2007;15(4):626-31. http://dx.doi.org/10.1590/S0104-11692007000400016

16. Acuña CVP, Gallardo AIC, Gonzáles VAM. Efectos de diferentes métodos de calentamiento utilizados en el perioperatorio en el adulto. Cienc. Enferm. 2009;15(3):69-75.

17. Brito MFP, Galvão CM. Os cuidados de enfermagem no uso da eletrocirurgia. Rev. Gauch. Enferm. 2009;30(2):319-27.

18. Poveda VB, Galvão CM. Hypothermia in the intraoperative period: can it be avoided? Rev Esc Enferm USP. 2011;45(2):411-17. http:// dx.doi.org/10.1590/S0080-62342011000200016 